\title{
Hans-Ulrich Treichel Und plötzlich wird ein Autor sichtbar
}

DOI 10.1515/iasl-2016-0005

\begin{abstract}
Many secondary educators, specifically academics in the humanities, are enormously productive authors and do not need to hide behind literary authors with respect to commitment and diligence. In contrast, academics avoid reflecting on their own writing practices as well as their methods in teaching it. A poetics of writing in the humanities has not been written until now, writing not being a regular part of academic curriculum, also because secondary educators recognize students more as researchers an academics but seldom as authors and writers.
\end{abstract}

\section{1}

Nur ein flüchtiger Blick in den Opac der Universitätsbibliothek Bielefeld oder auch Leipzig, und schon kann man unter dem Stichwort „Wissenschaftliches Schreiben" allein für den Zeitraum ab 2014 circa ein Dutzend Titel finden, die sich zumeist als Schreibratgeber für Akademiker verstehen. Offenbar besteht hier Beratungsbedarf. Wenn man in diese Bücher hineinschaut, kann man das Gleiche erleben wie auch bei der Lektüre von Creative-Writing-Büchern für das literarische Schreiben: dass sich hier durchaus vernünftige Ratschläge für die jeweilige Schreibpraxis finden und dass es eigentlich keinen Grund gibt, diesen Büchern mehr zu misstrauen als anderen auch. $\mathrm{Zu}$ meinen favorisierten und unvergessenen Ratschlägen aus einem solchen Ratgeber zählt beispielsweise die Aufforderung: Schreibe jeden Satz so, dass der Leser auch den nächsten lesen will. Dagegen ist weder aus literarischer noch aus wissenschaftlicher Sicht etwas zu sagen. Einen besseren Rat kann man gar nicht geben. Es fragt sich allerdings, wie dieser spezielle Satz jeweils aussehen soll. Subjekt-Prädikat-Objekt? Hypotaktisch oder parataktisch? Hegelianisch, nietzscheanisch, adornitisch? Mit nachgestelltem Reflexivpronomen? Schreiben wie Luhmann? Wie Derrida? Oder wie der eigene Doktorvater? Aber wie schreibt der eigentlich?

Die Schreibratgeber sind solange wahr, solange sie allgemein bleiben. Werden sie konkret, wird es oft banal oder aber kurios. Ein eher zufällig ausgewähltes

Kontaktdaten: Prof. Dr. Hans-Ulrich Treichel, Universität Leipzig, Deutsches Literaturinstitut Leipzig, Postfach 100920, D-04009 Leipzig, E-Mail: treichel@uni-leipzig.de 
neueres Beispiel stellt das Buch Erfolgreiches wissenschaftliches Schreiben von Simone Fischer dar, das insofern sogleich zur Lektüre einlädt, als es eben nicht nur zum richtigen wissenschaftlichen Schreiben verhelfen will, wie beispielsweise der Band Richtig wissenschaftlich schreiben: Wissenschaftssprache in Regeln und Übungen, ${ }^{1}$ sondern den Ratsuchenden auch zum erfolgreichen Schreiben verhelfen will. Wobei Schreiberfolg sich eben nicht nur in gutem Stil, sondern auch in Leistungspunkten, Zensuren, Stipendien und letztlich auch befristeten oder gar unbefristeten akademischen Arbeitsplätzen ausdrücken kann.

Auch Fischers Buch bietet keinen Anlass zur Polemik oder gar Häme. Ratschläge wie „Entspannt schreiben und bleiben“, „2,Den Einstieg finden“3 oder auch die „Die eigene Schreibbalance finden“ ${ }^{4}$ sind durchaus sinnvoll, erinnern allerdings an den Sportlehrer, der den Schülern „Schneller laufen“ und „Weiter springen" empfiehlt.

Auch die Frage „Wer ist hier der Autor - man, wir oder ich?“ ist hier am rechten Platz. Sie stellt sich in der Tat beim wissenschaftlichen Schreiben und muss vom Autor jeweils erneut beantwortet werden, seit das akademische „Wir“ seine Verbindlichkeit verloren hat und wir immer häufiger auch Ich-Sager unter den wissenschaftlichen Autoren finden. Des Öfteren kann bei diesen Ich-Texten freilich der Eindruck entstehen, dass das „Ich“ hier nicht ganz passend ist und ein „Wir“ womöglich angemessener wäre. Aber das ist vorerst nur ein Gefühl. Ganz sicher sind wir uns in dieser Sache nicht. Woher diese Unsicherheit rührt, wäre eine der Fragen, denen sich eine Poetik des wissenschaftlichen Textes zuwenden könnte. Oder ist die Frage nach Sprechweise des wissenschaftlichen Textes viel einfacher, als sie mir gerade vorkommen will? Hängt alles nur vom Rang des jeweiligen Autors ab? Darf der berühmte Wissenschaftler „ich“ sagen und der nicht berühmte nur „wir“? Die angemessene ,Sprechperspektive“ eines wissenschaftlichen Textes mag durchaus auch vom Ruhm oder der Präsenz eines Autors abhängen. Muss es aber nicht. Denn der eine sagt so und der andere möglicherweise anders.

Jürgen Habermas beispielsweise sagt „wir“. Zumindest in der 12. Vorlesung von Der philosophische Diskurs der Moderne oder auch im seinem Aufsatz Charles S. Pierce über Kommunikation. Adorno dagegen sagt weder ,ich“ noch „wir“, wie erste Stichproben - mit Hilfe der Datenbank Social Theory - ergeben. Zum Bei-

1 Helga Esselborn-Krumbiegel: Richtig wissenschaftlich schreiben: Wissenschaftssprache in Regeln und Übungen. 3. durchges. Aufl. Paderborn: Schöningh 2014.

2 Simone Fischer: Erfolgreiches wissenschaftliches Schreiben. Stuttgart: Kohlhammer 2015,

S. 156.

3 Fischer: Erfolgreiches wissenschaftliches Schreiben (Anm. 2), S. 160.

4 Fischer: Erfolgreiches wissenschaftliches Schreiben (Anm. 2), S. 162. 
spiel in dem Aufsatz Ist die Kunst heiter, in Versuch, das Endspiel zu verstehen oder auch im 3. Kapitel von Der getreue Korrepetitor. Den naheliegenden Scherz, dass er stattdessen „sich“ sagt, wollen wir uns verkneifen. Weitere Prüfungen wären notwendig, doch wird schon jetzt deutlich, dass der Ruhm des Autors nicht über seine Sprechweise entscheidet. Diese hängt meines Erachtens weitaus mehr vom Stoff wie auch von der Reflexionsdichte und dem Grad der Intertextualität des jeweiligen Textes ab. Ein eher persönlicher Essay mit nur wenigen Verweisen auf Sekundärliteratur verträgt durchaus eine selbstbewusste Ich-Instanz. Doch wenn wir beispielsweise mit Hilfe von vielen Primär- und noch mehr Sekundärzitaten über Thomas Manns Buddenbrooks oder Hegels Logik nachdenken, dann wäre es anmaßend, dieses von fremder Denk- und Schreibleistung durchwachsene Nachdenken unser eigenes zu nennen und für all das Ich-Originalität zu beanspruchen. Originalgenies unter Philologen sind selten und sehr persönlich schreibende Essayisten alles in allem auch. Zum Ausgleich dafür aber ist man als Literaturinterpret nie gänzlich allein. Was wir machen, hat immer auch schon jemand vor uns gemacht. Philologie ist denkende und schreibende Geselligkeit, so verschlossen oder gar verbarrikadiert das jeweilige Arbeitszimmer sein mag.

Zurück zu unserem Ratgeber. Simone Fischer beantwortet die Frage „Darf man ,ich“ schreiben?“ mit einem entschiedenen: „Ja, Sie dürfen!“ Das ist eine dankenswert eindeutige Antwort, die allerdings zwei Sätze später schon wieder eingeschränkt wird, wenn es heißt: „Sie sollten die Autorenreferenz in Hinblick auf die Ich-Form in jedem Fall vor Beginn Ihrer Arbeit mit Ihrem Betreuer besprechen." ${ }^{5}$

\section{2}

Womit ich bei den Betreuern wäre. Den Dozenten und Hochschullehrern also, die in der Regel Vielschreiber sind. Zum einen, weil sie es müssen und zum anderen, weil sie es wollen. Schreiben muss man um der Laufbahn willen. Kein Doktortitel ohne Doktorarbeit und keine Berufung zum Professor ohne eine ansehnliche Publikationsliste. Mindestens zwei Monographien und ein oder zwei Dutzend ,selbstgeschriebene' Aufsätze sollten es schon sein. Manchmal ist auch weniger möglich, oft genug ist es auch mehr. Es finden sich unter Hochschullehrern enorm produktive Menschen, vom Schreiben geradezu besessene, die immerzu ,an irgendwas sitzen'. Zumeist an einem Aufsatz, oft genug auch an einem Buch. Obwohl sie es ja gar nicht mehr müssten. Wer erst einmal verbeamtet ist, könnte

5 Fischer: Erfolgreiches wissenschaftliches Schreiben (Anm. 2), S. 148. 
dem Schreiben abschwören. Was auch gelegentlich vorkommt, aber nicht die Regel ist. Die Regel ist eher unaufhörliches Schreiben, beständig fortgesetzte Produktivität, ein Aufsatz nach dem anderen.

Bisher ist noch niemand darauf gekommen, in solch einer beispielsweise germanistischen Produktivität eine Form von blindem Drang oder gar schöpferischer Besessenheit zu sehen. Emphatische Produktivitätskategorien dieser Art sind bisher nur den Schriftstellern gegönnt worden. Dem Mythos des prometheischen Schriftstellers hat sich kein Mythos des prometheischen Germanisten beigesellt. Und nicht nur das. Gelegentlich kommt es sogar zur Verachtung dieser Art von Produktivität, wenn beispielsweise ein George Steiner das Wuchern des Sekundären beklagt, als handle es sich beim Schreiben von literaturwissenschaftlichen Texten nicht um geistige Aktivität und schriftliche Reflexion von Lektüre, sondern um einen krankhaften Prozess. Was nicht zuletzt auch deshalb ungerecht ist, weil die jeweilige Anstrengung, das Opfer an Zeit, die notwendige soziale Isolierung, die verdorbenen Familienferien, bei einem Autor von philologischen Aufsätzen ebenso dramatisch sein kann wie bei einem Autor von Romanen. Man schaue sich nur einmal die geradezu überbordenden Schriftenverzeichnisse so mancher dieser akademischen Autoren an: schriftgewordene Leben sind das, die Zurücknahme des Lebens zugunsten der Schrift, die Wortwerdung des Fleisches, wie bei Kafka, nur dass dieses Schreiben eben keinen Prozess, kein Schloß und keine Verwandlung hervorbringt, sondern gegebenenfalls einen weiteren Aufsatz über das Schloss, den Prozess und die Verwandlung. Und dies ganz unbeeindruckt davon, dass in der Internationalen Kafka-Bibliographie von Maria Luise Caputo-Mayr und Julius M. Herz allein die kommentierte Bibliographie der Artikel und kleineren Beiträge, auch Aufsätze in unserem Sinne zählen dazu, einen Umfang von 451 Druckseiten ausmacht. ${ }^{6}$ Wobei der Zeitraum von 1997 bis heute hier noch gar nicht erfasst ist.

\section{3}

Psychodynamisch, vom nie zu beruhigenden Schreibimpuls und von der Lebenspraxis her mögen sich die Anstrengungen von wissenschaftlichen und literarischen Autoren also durchaus gleichen. Der jeweilige Profit ist aber in der Regel ein anderer: Hier Ruhm - und hoffentlich auch Geld. Und dort Verbeamtung -

6 Vgl. Marie Luise Caputo-Mayr/Julius Michael Herz: Franz Kafka. Internationale Bibliographie der Primär- und Sekundärliteratur 1955-1997. Bd. 2: Kommentierte Bibliographie der Sekundärliteratur 1955-1997. München: Saur 2000, S. 63-514. 
und immer Geld. Zumindest im jeweils besten Falle. Eine weitere Differenz betrifft die Praxis der Selbstreflexion. Literarischen Autoren ist das eigene Metier und das Schreiben sehr oft ein Gegenstand des Nachdenkens. Und auch des Darüberschreibens. Man denke nur an die Poetikvorlesungen, in denen sich ein Autor in aller Öffentlichkeit der eigenen Schreibpraxis zuwendet. Wo er keine Gedichte, keine Erzählung vorliest, sondern über sein Schreiben von Lyrik oder Prosa nachdenkt, als wäre er sein eigener Germanist. Und manchmal ist er es auch, wenn es ihm gelingt, wie es beispielsweise Thomas Mann mit seinem Buch Die Entstehung des Doktor Faustus gelungen ist, einen Sekundärtext über sich selbst kanonisch werden zu lassen, so dass kein Philologe mehr unbeeinflusst davon bleibt, wenn er sich mit dem Autor beschäftigt.

Für Schriftsteller ist es allerdings nichts Besonderes, über das eigene Schreiben nachzudenken. Das Schreiben fordert es heraus, und es gehört zum Schreiben dazu. Denn es ist eine konfliktreiche und schwierige Tätigkeit, und je mehr man schreibt, um so mehr wird einem die Sprache zum Problem. Ein Schriftsteller ist jemand, dem das Schreiben besonders schwer fällt, heißt es sinngemäß in Thomas Manns Tristan, und nicht wenigen Autoren läuft in den Phasen ihrer schriftstellerischen Entwicklung die Muttersprache erst einmal davon, um dann erneut wieder angeeignet werden zu müssen. Beständig droht das Scheitern, beständig droht der Souveränitätsentzug. Das sind Erfahrungen, die sich nur ertragen lassen, wenn man wiederum über sie schreibt, um sie, wenn nicht zu verstehen und beherrschbar zu machen, so doch wenigstens als Reflexions- und Erzählstoff zu nutzen.

Philologen sind davon anscheinend unberührt. Auch der besessenste Autor von Aufsätzen, auch der Germanist mit dem umfangreichsten aller Publikationsverzeichnisse schreibt nicht über sein eigenes Schreiben. Warum eigentlich nicht? Schließlich ist es doch für ihn nicht nur berufliche Tätigkeit, sondern ein ,Existential', dem er einen guten Teil seiner Lebenszeit und seiner produktiven Kräfte widmet. Eine Antwort auf die Frage nach dem Grund der Zurückhaltung in eigener Sache könnte Bescheidenheit sein, eine Haltung des Hinter-die-SacheZurücktretens. Und die Sache ist das Fach und ist das jeweilige Werk oder der Autor, über den man schreibt. Man ist ja schließlich keiner dieser Künstler, die sich mit ihren Angelegenheiten immer an den Bühnenrand drängen. Man gehört, um im Bild zu bleiben, eher zum technischen Personal, also hinter die Bühne oder dorthin, wo die Beleuchter sind. Eine weiterer Grund wäre vielleicht der, dass es sich bei wissenschaftlichen Texten eben um eine pragmatische Textsorte handelt. Um Texte, die nicht brillant sein müssen, sondern nur gut genug, um ihren Zweck des wissenschaftlichen Beitrags zu erfüllen und bei denen sich Stilfragen oder Fragen der Dramaturgie oder gar des ,Plots‘ eben nur ex negativo stellen, nur dann, wenn der Text deutliche stilistische Schwächen zeigt oder schlecht gebaut 
ist. Ansonsten reicht es, fehlerfrei, lesbar, klug und gegebenenfalls innovativ zu sein. Wobei Letzteres aber nur im engeren Rahmen möglich ist, denn man dreht nie allein am gleichen Rad oder der gleichen Schraube.

Mit diesen Antworten wäre der Frage nach der Praxis und Poetik des wissenschaftlichen Schreibens jede Dringlichkeit genommen, wenn sich nicht zugleich der Gedanke aufdrängte, dass ein brillanter Text oder auch einer, der zumindest mehr als gut genug ist, zugleich der wissenschaftlich wertvollere ist. Die Frage nach dem Verhältnis von Wissen und Poesie wäre auf unseren Kontext bezogen die nach dem Zusammenhang von Stil und Wissen auch im Sachtext. Und schließlich: Die meisten wissenschaftlich Schreibenden sind auch wissenschaftlich Lehrende. Speziell in den Geisteswissenschaften aber sind die Lernergebnisse immer an die Schriftlichkeit, die schriftliche Leistung gebunden. Die Hochbegabten der Mündlichkeit, wenn es sie denn geben sollte, und wie sie zuweilen in Unterrichtsformen wie dem Problem-based Learning ihre Chance erhalten, sind am Ende die Blamierten, wenn sie ihr Wissen und ihre rhetorischen Fähigkeiten nicht auch schriftlich überzeugend dokumentieren. Nur wer schreibt, der bleibt zumindest an der Universität.

\section{4}

Noch ist es keine Selbstverständlichkeit an unseren Universitäten, dass nonfiction-writing-Seminare das Studium begleiten und Schreibkompetenz ein in den Studienordnungen vorgegebenes Lernziel ist. Noch wird gegen die Regel „Was geprüft wird, muss auch gelehrt werden“, fortwährend verstoßen. Genauso wenig wie es eine Selbstverständlichkeit ist, dass die Wissenschaftler ihre Schreibwerkstätten öffnen und die Studierenden beim Schreiben zusehen lassen. Besonders diejenigen Wissenschaftler, die als gute Autoren gelten, seien es Geistes-, Sozialoder auch Naturwissenschaftler. Auch letztere müssen schreiben, wenn auch unter anderen Vorzeichen und nicht selten im Kollektiv. Wobei die Verschlossenheit des Schreibvorgangs kein moralischer Vorwurf gegen die Wissenschaftlerzunft sein soll. Die Werkstätten bleiben unseres Erachtens nicht verschlossen, weil die Goldmacher ihre geheimen Rezept nicht verraten wollen. Der Grund ist wohl eher der, dass diejenigen, die gut schreiben, ihr eigenes Schreibgeheimnis, ihre eigenen stilistischen und dramaturgischen Verfahren, Melodie und Rhythmus ihrer Texte, Spannungsaufbau der Argumentation, Dramaturgie der Zitate, die ,Erzählung der Recherche“ - dass sie also ihre eigenen Schreibverfahren gar nicht so genau kennen, wie es den Anschein hat. Sie tun es, aber sie wissen es nicht. Vieles geschieht eben intuitiv, wie im literarischen Schreiben auch. Und die 
implizite Poetik des eigenen Tuns bleibt den meisten Wissenschaftsautoren wohl eher verborgen. Bisher hat ja auch niemand danach gefragt.

Was insofern überraschend ist, als es sich hierbei, wir sprechen ja vor allem von Literaturwissenschaftlern, um Spezialisten auch in eigener Sache handeln sollte. Die Schnittstellen zwischen literarischen und wissenschaftlichen Schreibweisen sind zudem durchaus erkennbar. Auch ein Aufsatz ist oder sollte komponiert sein. Auch ein Sachtext ist letztlich persönlich und hinsichtlich des Sprachgefühls, der Stoffwahl, des Tonfalls, des entschiedenen oder auch weniger entschiedenen argumentativen Zugriffs autobiographisch grundiert. Sonst wären ja alle Sachtexte gleich. Wenn Tolstoi sagt, alle glücklichen Familien ähneln einander, nur die unglücklichen unterscheiden sich, dann ließe sich für die Qualität von Texten möglicherweise umgekehrt folgern: die Brillanz von Texten ist jeweils einzigartig, allenfalls die Schwächen sind gleich, wenn Texte sich beispielsweise als begriffslastig, sperrig, von Zitaten beschwert oder von der Dramaturgie der Argumentation her als spannungsarm erweisen. Das oft beklagte entindividualisierte Grau in Grau der Sekundärliteratur dürfte hierin ihren Grund haben. Wo aber dies nicht so ist, zeigt der Text Konturen und individuelles Profil. Und plötzlich wird ein Autor sichtbar.

Und wenn dieser Autor schon sichtbar wird, und dieses auch ohne „ich“ zu sagen, dann sollte er sich, als Dozent oder Hochschullehrer, auch seinen Studenten als Autor zeigen. Denn die wenigsten wissen, dass dort am Pult einer steht, der nicht nur viel weiß und vieles prüft, sondern zu Hause am Schreibtisch gegebenenfalls die gleichen Mühen hat wie sie selbst. Nur eben mehr Erfahrung. Die aber ließe sich, das Wissen um die eigene Praxis vorausgesetzt, auf gewinnende Weise teilen. 